\title{
Vulvovaginectomy and neo-urethrostomy for treatment of haemangiosarcoma of the vulva and vagina
}

\author{
T P Hill ${ }^{\mathrm{a}}, \mathrm{R}$ G Lobetti ${ }^{\mathrm{b}}$ and M L Schulman ${ }^{\mathrm{c}}$
}

\begin{abstract}
Vulvovaginectomy and neo-urethrostomy were performed in a 9-year-old German shepherd dog following a diagnosis of infiltrative vulvar and vestibulovaginal haemangiosarcoma. The dog was presented for intermittent vulvar haemorrhage over a 3-month period. On examination the vulva and vestibulovagina were distended and firm. Vaginal discharge and fine needle aspiration cytology detected anaplastic cells. Haemangiosarcoma was diagnosed on biopsy. A new urethral opening was created in the floor of the vagina allowing resection of the vulva and caudal vestibulovagina. Urinary continence was preserved and healing was without complications.
\end{abstract}

Key words: urethrostomy, vaginal, vulvar, vulvovaginectomy.

Hill T P, Lobetti R G, Schulman M L Vulvovaginectomy and neo-urethrostomy for treatment of haemangiosarcoma of the vulva and vagina. Journal of the South A frican Veterinary Association (2000) 71(4): 256-259 (En.). Department of Companion Animal Surgery, Faculty of Veterinary Science, University of Pretoria, Private Bag X04, Onderstepoort, 0110 South Africa.

\section{INTRODUCTION}

Primary tumours of thevulva and vagina account for 2-3\% of neoplasms in dogs $5^{2,7,8}$. Most are benign, with leiomyomas and fibromas being most common ${ }^{2,7}$. Leiomyosarcoma and carcinoma are infrequently-found malignant tumours ${ }^{2,4,7,8}$ whereasthetransmissiblevenereal tumour (TVT) is a more common tumour, especially in resource-poor communities in South Africa.

Tumor resection, commonly facilitated by dorsal episiotomy, is successfully used to treat benign tumours ${ }^{2,7}$. Vulvectomy for malignant tumours has been described, but tumour recurrence is common ${ }^{7}$, and patients with tumours deemed inoperableare often euthanased ${ }^{5}$. More extensive vulvovaginectomy and perineal urethrostomy has been described for infiltrative tumours of this region ${ }^{1}$. Haemangiosarcoma of the vulva and vestibulovagina in the dog is rare, with only a single case reported previously ${ }^{7}$.

The purpose of this report is to describe the clinical manifestations of a genital tract haemangiosarcoma and surgical treatment performed, as compared to

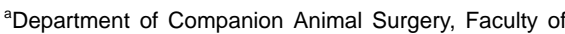
Veterinary Science, University of Pretoria, Private Bag X04, Onderstepoort, 0110 South Africa.

${ }^{b}$ Department of Companion Animal Medicine, Faculty of Veterinary Science, University of Pretoria.

Department of Reproduction, Faculty of Veterinary Science, University of Pretoria.

${ }^{*}$ Author for correspondence.

Received: June 2000. Accepted: August 2000. previously published descriptions of treatment of malignant tumours in the vulval area.

\section{CASE HISTORY}

A 9-year-old female German Shepherd Dog was referred to the Onderstepoort Veterinary Academic Hospital (OVAH) with a history of intermittent vulvar haemorrhage of approximately 3 months' duration. A diagnosis of pyometra had been made 6 months before referral and treated by ovariohysterectomy. On presentation at the OVAH, the dog was pyrexic $\left(40.5^{\circ} \mathrm{C}\right)$, lethargic, underweight and showed discomfort when attempting to sit. The vulva and perineum appeared enlarged and prominent with a continuous haemorrhagic discharge (Fig. 1). On pal pation of the perineum, the vulva and vestibulum were hard and non-elastic and it was not possible to determine whether the enlargement was caused by an intramural or intraluminal mass. Both popliteal and inguinal lymph nodes were

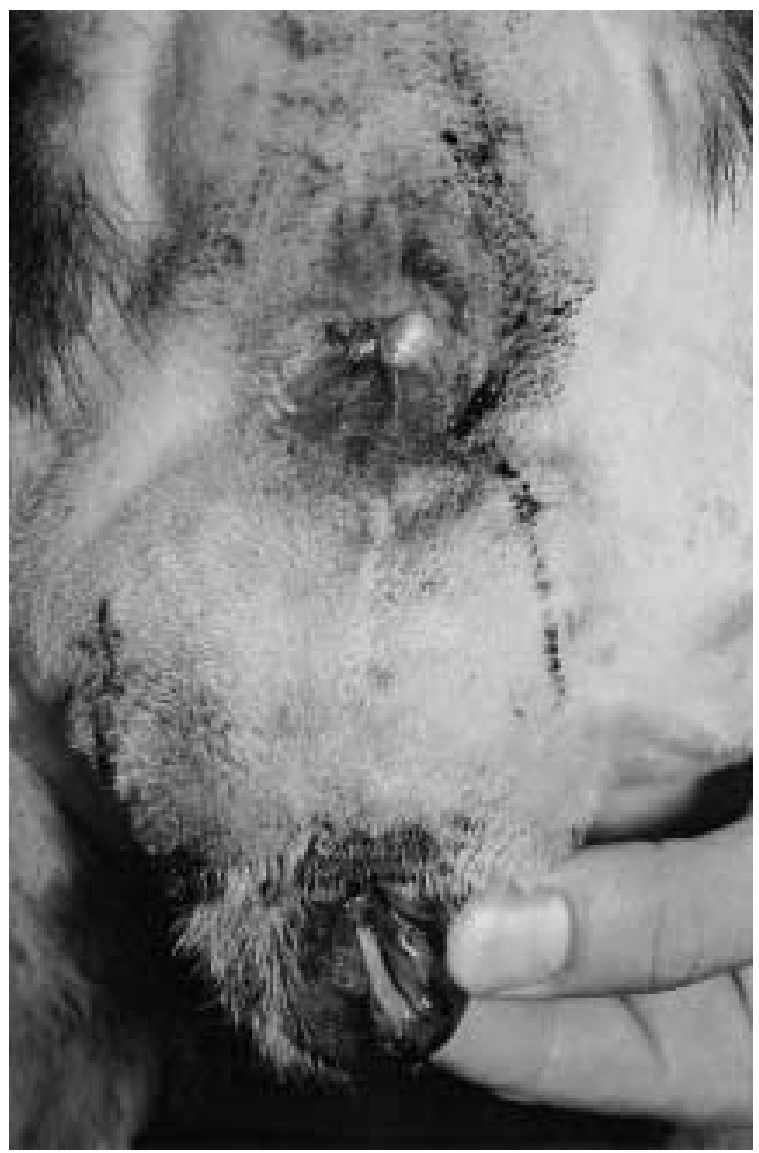

Fig. 1: Enlarged distended vulva and caudal vagina. There is also a surgical wound on the left-hand side, which represents the initial biopsy site. 
normal on palpation. Urination produced a strong urine flow with blood clots present in the initial part of the urine stream.

On digital examination, the vestibular and caudal vaginal lumen was very narrow as a result of a large intramural mass compressing the vaginal wall. The mass was approximately $7 \mathrm{~cm}$ in size and occupied the entire floor and right wall of the vulva, vestibulumand caudal vagina. The narrow vaginal lumen and ongoing haemorrhage hindered vaginoscopic examination. Numerousblood clots and a large erythematous area were observed on the vestibular floor. Multiple areas of petechiae and ecchymoses were present throughout the vestibular and caudal vaginal mucosa as well as small ulcerations over the mass. The urethral opening could not be visualised.

Haematology revealed marked leucocytosis (30.1 × 10\% $/ l$, normal 6-15), mature neutrophilia ( $24.98 \times 10^{9}$, normal 3.0-11.5) and monocytosis (1.51 × $10^{9}$, normal $0.15-1.35)$. Serum chemistry was unremarkable. A urine sample collected by cystocentesis appeared normal macroscopically and had a pH of 7 and specific gravity of 1.025 . Moderate proteinuria, haemoglobin crystalluria and few erythrocytes were detected on urinalysis. Smears obtained from the vulvar discharge for cytological examination were highly cellular, with obvious secondary infection and numerous anaplastic cells. However, no specific tumour diagnosis could be made. Fine needle aspirates of the mass demonstrated solitary and aggregated anaplastic cells. A presumptive diagnosis of anaplastic sarcoma was made.

Thoracic radiographs for lung metastases revealed no significant changes. Abdominal ultrasound showed a $2 \mathrm{~cm}$ soft tissue mass dorsal to the bladder trigone. It was composed of two adjacent tubular structures sharing a common wall and was considered to be the remnants of the previously ligated uterine horns and body. Ultrasound of the perineum confirmed a large soft tissuemass on thefloor and right side of the vestibulovagina extending up to the ischial rim. The margins of the tumour were clearly demarcated and did not appear to extend into the pelvic canal.

An incisional biopsy wastaken from the left lateral aspect of the vaginal mass. Areas of necrosis, fibrosis, haemorrhage and neutrophil infiltration werefound on histopathological examination. Nests of anaplastic cells with a high mitotic index were found in the necrotic areas and in some instances formed ill-defined vascular channels. A diagnosis of anaplastic, infiltrative haemangiosarcoma of the vagina was made. Echocardiography

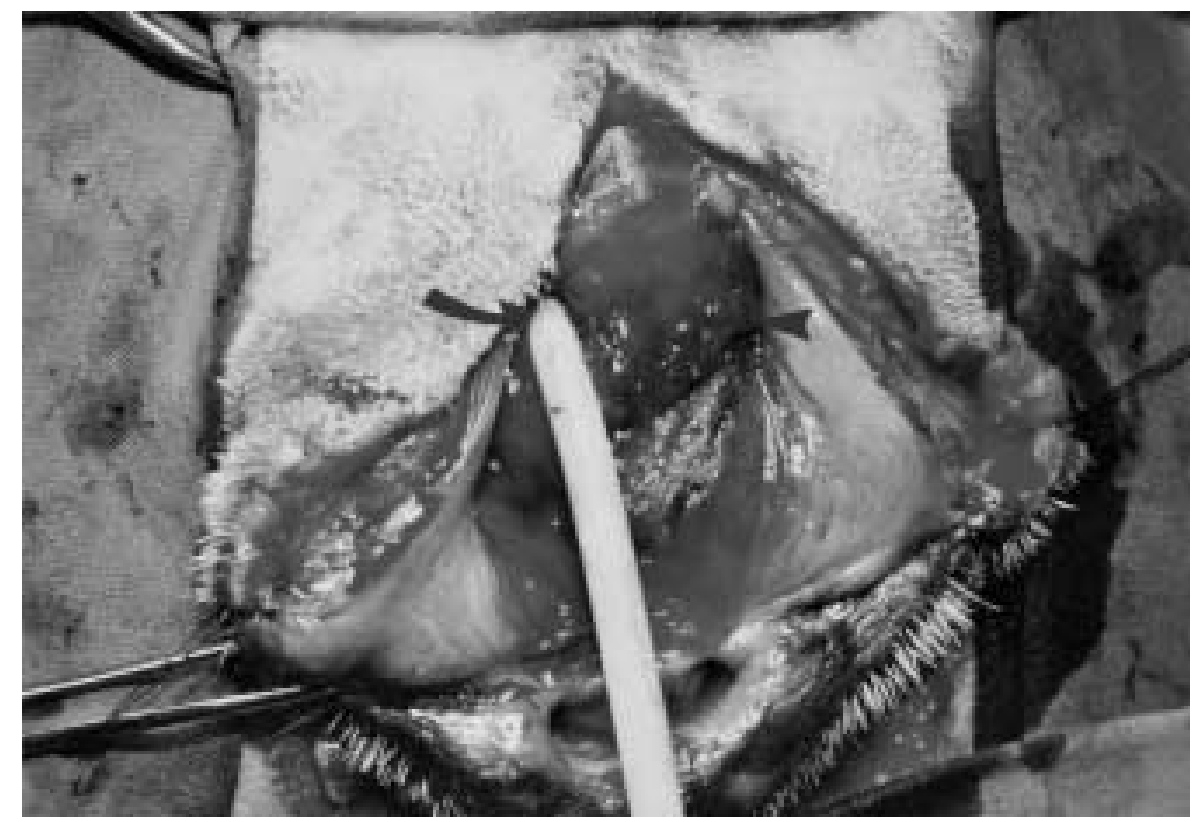

Fig. 2: Dorsal episiotomy - the external urethral is catheterised (curved arrow) and displaced by the haemangiosarcoma (arrowhead). revealed no evidence of atrial metastases.

The perineal area was surgically prepared in astandard manner and included a purse-string suture of theanus. Theanimal was placed in sternal recumbency with the perineum elevated. Theurethral opening wasidentified following a dorsal episiotomy. It was displaced dorsolaterally to the right and recessed within thetumour (Figs 2, 3). A 16F Foley catheter (Macmed) was placed to drain the bladder and identify the urethra. A fusiform

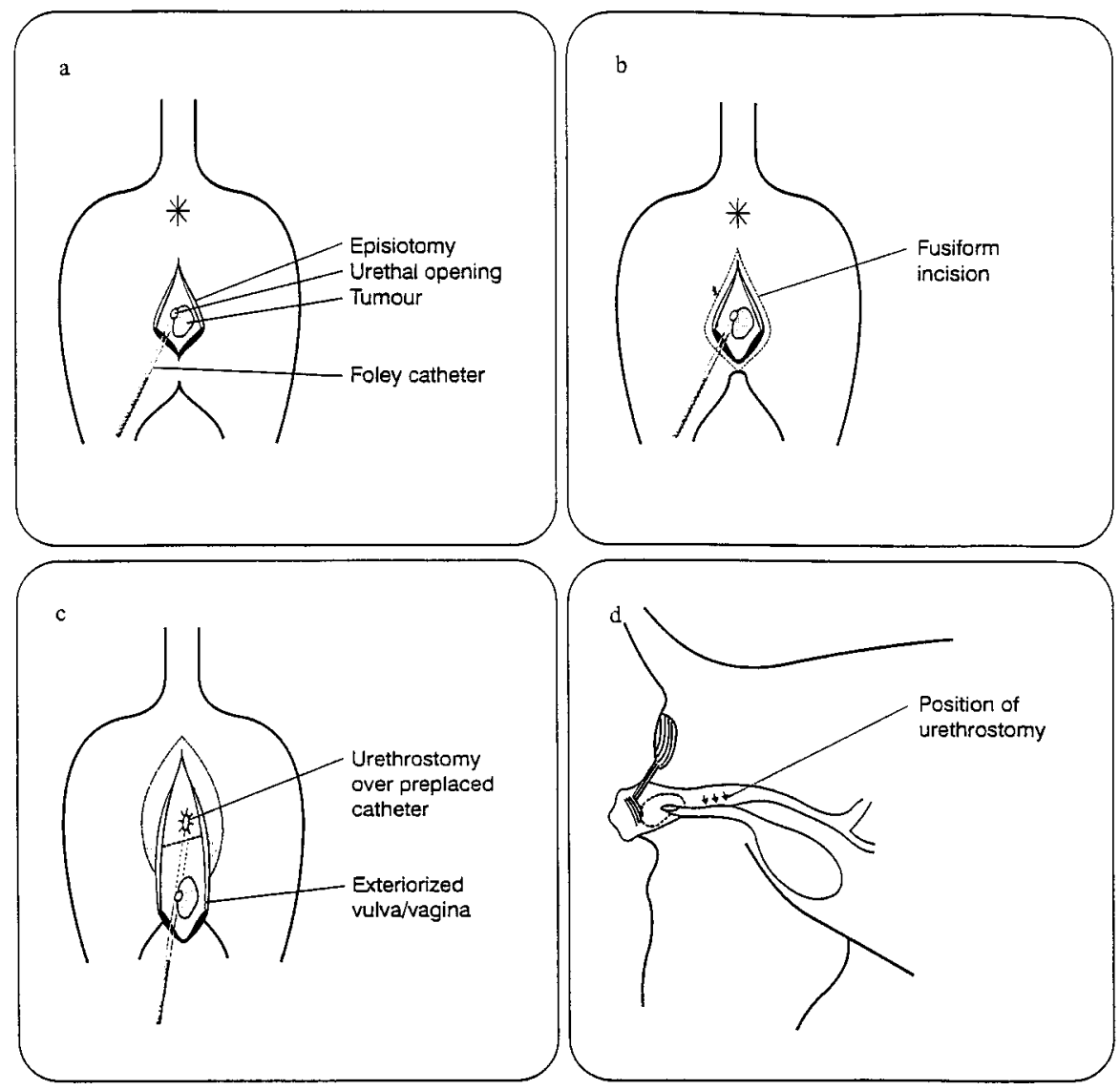

Fig. 3: Schematic presentation of surgical excision. a: Episiotomy to visualise tumour and urethral opening; b: fusiform incision to include vulva, episiotomy and tumour; c: exteriorised vulva and vagina, urethrostomy over catheter; $d$ : position of urethrostomy in floor of vagina. 
skin incision was madearound the vulva, vestibulum and vagina so as to encompass the ventral vulval commissure, the palpable portion of the vestibulum and vagina dorsally, the previous biopsy site and theepisiotomy incision. The constrictor muscle of the vestibulum was transected below the junction with the external anal sphincter. The ischiocavernosus and ischiourethralis muscles were detached from the ischial rim. Branches of the ventral perineal artery were ligated or electrocoagulated as encountered.

The cranial extent of the tumour was identified through the episiotomy incision. The Foley catheter/urethra was palpated and identified. A $2 \mathrm{~cm}$ longitudinal incision was made over the catheter $2 \mathrm{~cm}$ cranial to edge of the tumour. The longitudinal incision extended through the vaginal mucosa, vaginal and urethral muscleand urethral mucosa to expose the intramural intrapelvic portion of the urethra. The urethral mucosa was sutured to the vaginal mucosa with 5/0 simple interrupted polydioxanone (PDSII, Schering-Plough) sutures and a new urethral opening cre ated. The vulva, vestibulum, caudal vagina and distal part of the urethra, together with the tumour were resected immediately caudal to the neourethrostomy site. A small amount of tumour tissue was identified within the vaginal muscle layer on the left periphery. An additional $2 \mathrm{~cm}$ section of the vagina was resected obliquely on theleft. Subcutaneoussutures using $3 / 0$ polydioxanone were placed to eliminate dead space and eliminate skin tension. Theskin was sutured to the muscular and mucosal layers of the vagina using 4/0 polydioxanonein a simpleinterrupted pattern (Fig. 4).

Histopathological examination of the excised tumour confirmed the incisional biopsy results. Further immunoperoxidase staining was positive for Von Willebrand factor, confirming a haemangiosarcoma. Although thelateral margins of the surgical field were free from neoplastic cells, thedeeper portion of thesurgical excision contained neoplastic cells.

Post-operatively, the dog was given $0.01 \mathrm{mg} / \mathrm{kg}$ buprenorphine (Temgesic, Schering-Plough), repeated 8 hours later. Thereafter, ketoprofen (Orucote, Rhône Poulenc Rorer) at $1 \mathrm{mg} / \mathrm{kg}$ once a day for 2 days and amoxycillin/clavulanic acid (Synulox, Pfizer) at $20 \mathrm{mg} / \mathrm{kg}$ twice a day for 10 days wereadministered. Petroleum jelly was applied topically around the new vaginal opening. A strong urineflow was present but urination was uncomfortable for the dog for the first 24 hours. No signs of urinary incontinence were noted. Further post-surgical recovery was

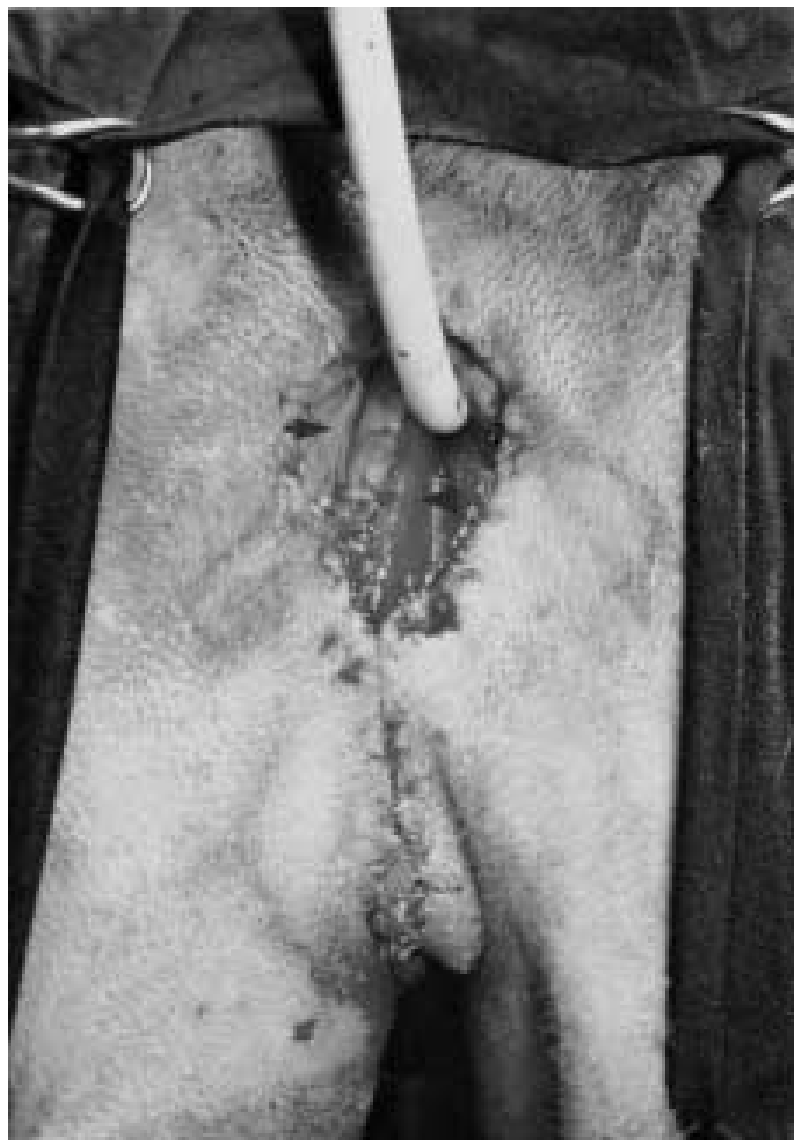

Fig. 4: Spatulated urethra with the urethral mucosa (arrowhead) sutured to the vaginal mucosa (arrow) of the caudal vagina.

uneventful. The owner dedined adjunct chemotherapy with doxorubicin.

Five months post-operatively, signs of vaginal bleeding returned and tumour recurrence was diagnosed on clinical examination. The dog was euthanased at the request of the owner. N o post mortem examination was performed.

\section{DISCUSSION}

Primary tumours of the vulva and vaginaareusually (71-82\%) benign $n^{2,7}$. In a report by Kydd and Burnie ${ }^{5}$, who examined 21 cases of vulvar and/or vaginal neoplasia histopathologically, 20 of the 21 cases were found to be benign. Apart from leiomyomas and fibromas, other types of benign neoplasia reported are lipoma, histiocytoma, benign melanoma and myxoma ${ }^{7}$. Malignancy accounts for only a small percentage of vulvar and/or vaginal tumours, particularly if TVT is excluded $^{2,7}$. TVT alone accounts for be tween $37 \%$ and $60 \%$ of malignant tumours ${ }^{2,7,8}$. Other common malignant tumours are leiomyosarcoma and squamous cell carcinoma ${ }^{2,7,8}$. Only single cases of mast cell tumour, epidermoid carcinoma, haemangiosarcoma, osteosarcoma and adenocarcinoma have been reported ${ }^{1,2,7}$. Magneand others ${ }^{6}$ reviewed 7 cases that presented with avaginal and/or vestibular mass that resulted from a caudal extension of a bladder or urethral carcinoma.

The most common clinical signs of vulval/vaginal tumours are an abnormal vulvar discharge or the sudden appearance of a protruding mass. Many of the benign tumours are pedunculated, grow within theconfines of the vaginaand only at a later stage protrude through the vulval lips ${ }^{7,8}$. Leiomyomas originate from the smooth muscle of the vagina or vestibulum and may be intramural or extraluminal ${ }^{8}$. Other presenting signs may be vulval or perineal swelling, dysuria and tenesmus ${ }^{5}$. Urinary incontinence does not occur ${ }^{5}$. The average age on presentation depends on the type of tumour. In general, dogs with TVT present at younger age (average age of 4.4 years), whereas dogs with other tumours present at a much older age (10.8-11.2

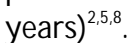

Most malignant tumours are nonpedunculated ${ }^{7}$. Vulvectomy has been described as a treatment for malignant tumours, but recurrence is common ${ }^{7}$. In some cases, tumours have been deemed inoperable following exploratory episiotomy, and the animal subsequently euthenased ${ }^{5}$. The averagesurvival timeis approximately $30 \%$ shorter than those with benign tumours ${ }^{7}$. A more extensive surgical resection technique combining 
vulvovaginectomy and perineal urethrostomy has been described for large and infiltrative tumours of this region ${ }^{1}$.

This case of vaginal haemangiosarcoma in the dog is only the second published case ${ }^{7}$. In the previously-reported case, the tumour was incompletely excised with rapid regrowth of the tumour within 1 month ${ }^{7}$. Although thetumour described in this report was incompletely excised, tumour regrowth occurred only 5 months post surgery.

The surgical technique described here avoids creating a perineal urethrostomy, but rather creates a neo-urethrostomy site within the remaining proximal portion of the vagina. Deep dissection of the pelvic urethra with possible damage to the nearby caudal vesicular artery, ureters, pelvicplexusand pudendal nervebranches is thus avoided. It is possible that the undisturbed longitudinal musclefibres of the vaginal and urethral muscle immediately surrounding the new urethrostomy site may be helpful in compressing the opening and preventing retrograde urethritis and cystitis. Sutures between the vaginal and urethral mucosa were without tension. Suturing the urethra directly to skin requires caudal traction of the ure thra and is morelikely to result in tension on the suture line. Excessive suture line tension resulting in wound dehiscence is a common cause of urethral stricture formation in perineal urethrostomies in cats $^{3}$. A disparity in the thickness of the tissue edges was not experienced.

With a neourethrostomy, urineis directed onto vaginal mucosa rather than skin, which may reduce problems with urine scald. The remaining portion of the vagina, cervix and uterus remains connected to the outside. Also, the peritoneal cavity is not entered. Dissection of the urethral stump or removal of the uterine remnants may cause penetration of the peritoneal cavity and allow seeding of neoplastic cells or infection into the abdomen. It is also possible that urovagina may occur if the neo-urethrostomy opening lies dorsal to the ischial arch. To prevent this possible complication the neo-urethrostomy opening must be caudal to the ischial arch.

Vulvovaginectomy and neo-urethrostomy may be curative for large benign tumours involving the vulva and vagina of the bitch with minimal post surgical complications such as urinary incontinence, retrograde urethritis and urine scald. A similar result may be possible with early malignant tumours but palliation may still be achieved for advanced malignancies, as was shown in this case.

\section{ACKNOWLEDGEMENTS}

Professor Robert Kirberger performed the diagnostic imaging and Drs Emily Lane and Joyce Pearson performed the histopathology examination. Their contribution to this case is gratefully acknowledged.

\section{REFERENCES}

1. Bilbrey SA, Withrow SJ, Klein M K, Avery Bennett R, N orris A M, Gofton N, DeH off W 1989 Vulvovaginectomy and perineal urethrostomy for neoplasms of the vulva and vagina. Veterinary Surgery: 18: 450-453

2. Brodey R S, Roszel J F 1967 Neoplasms of the canine uterus, vagina, and vulva: a clinicopathologic survey of 90 cases. Journal of the American Veterinary M edical Association: 151: 1294-1307

3. Hosgood G, Hedlund C S 1992 Perineal urethrostomy in cats. Compendium of Continuing Education for the Practising Veterinarian 14:1195-1205

4. Jubb K V F, Kennedy P C, Palmer N 1993 Pathology of domestic animals (4th edn). Academic Press, San Diego: 450-454

5. Kydd D M, BurnieA G 1986 Vaginal neoplasia in the bitch: a review of forty clinical cases. Journal of Small Animal Practice 27: 255-263

6. Magne M L, Hoopes PJ, Kainer R A, Olson $P N$, Husted P W, Allen T A, Wykes P M, Withrow SJ 1985 Urinary tract carcinomas involving the canine vagina and vestibule. Journal of the American A nimal H ospital A ssociation 21: 767-772

7. Thacher C, Bradley R L 1983 Vulvar and vaginal tumours in the dog: a retrospective study. Journal of the American Veterinary M edical A ssociation 183: 690-692

8. Withrow SJ, Susaneck SJ. 1986 Tumours of the canine female reproductive tract. In Morrow D A (ed.) Current therapy in theriogenology (2nd edn). W B Saunders, Philadelphia: 525-527 